\title{
A persistent neutrophil-associated immune signature characterises post-COVID19 pulmonary sequelae
}

\section{Peter George}

Imperial College London

Anna Reed

Imperial College London

Sujal Desai

Imperial College London

Anand Devaraj

Imperial College London

Tasnim Shahridan Faiez

Imperial College London

\section{Sarah Laverty}

Imperial College London

Michael Liu

Imperial College London

Faisal Kamal

Royal Berkshire Hospital

William Man

Imperial College

Sundeep Kaul

Imperial College

\section{Suveer Singh}

Imperial College London

Georgia Lamb

Imperial College London

Thomas Burgoyne

University College London

Andreia Pinto

Royal Brompton and Harefield Clinical Group

\section{Hugo Farne}

Imperial College

Alessia Dalla Priya 
Imperial College

\section{Andrew Aswani}

Guy's and St Thomas' NHS Foundation Trust

\section{Francesca Patella}

Kinomica Ltd

\section{Weronika Borek}

Kinomica Ltd

\section{Arran Dokal}

Kinomica Ltd

\section{Xiao-Ning Xu}

Imperial College

\section{Peter Kelleher}

Imperial College London https://orcid.org/0000-0002-4392-5527

\section{Anand Shah}

Imperial College

Aran Singanayagam ( $\nabla$ a.singanayagam@imperial.ac.uk)

Imperial College London https://orcid.org/0000-0001-9849-0033

\section{Article}

\section{Keywords:}

Posted Date: February 25th, 2022

DOI: https://doi.org/10.21203/rs.3.rs-1293175/v1

License: (c) (1) This work is licensed under a Creative Commons Attribution 4.0 International License.

Read Full License 


\section{Abstract}

Persistent interstitial lung changes with associated symptoms occur in a proportion of individuals that have recovered from severe acute respiratory syndrome coronavirus-2 (SARS-COV-2) infection through unknown mechanisms. We studied individuals with severe COVID-19 longitudinally following recovery from acute illness. Subjects with interstitial lung changes at 3-6 months post-recovery had an upregulated neutrophil-associated immune signature including increased chemokines, proteases and markers of neutrophil extracellular traps detectable systemically. Similar pathways were enriched in the upper airway with a concomitant augmentation of antiviral type-I interferon signalling. Interaction analysis of the peripheral phosphoproteome identified enriched kinases critical for neutrophil inflammatory pathways. Repeat sampling indicated that full normalisation of radiological and functional changes has not yet been reached in many individuals by 12 months post-recovery. These data provide functional insight into mechanisms driving pulmonary sequelae of COVID-19 and provide a rational basis for development of future targeted approaches to prevent long-term complications.

\section{Introduction}

Clinical presentation following infection with severe acute respiratory syndrome coronavirus 2 (SARSCoV-2) is broad ranging from a mild self-limiting illness to severe respiratory failure requiring intensive support ${ }^{1}$. Numerous studies have assessed the early acute phases of coronavirus disease 2019 (COVID19) but there has been relatively less focus on the longer term sequelae induced by this novel infectious disease. The majority of affected individuals make a full clinical recovery but a significant proportion experience long term health consequences and a diverse range of symptoms have been described as part of the post-COVID syndrome ${ }^{2-4}$. Persistent radiological abnormalities associated with pulmonary function impairment occur in some infected individuals ${ }^{5-8}$ but the pathogenic mechanisms underlying this downstream consequence are poorly characterized.

Clinical studies reveal that patients with severe COVID19-related acute respiratory distress syndrome (ARDS) are at greatest risk of pulmonary complications ${ }^{9,10}$. However, the reasons why some individuals make a full clinical, physiological and radiological recovery whilst others follow a more perilous trajectory with persistent interstitial lung changes and associated pulmonary function impairment are poorly understood. Acute severe COVID19 is characterised by hyperinflammation detectable both systemically and within the airways ${ }^{11-13}$ and there is growing evidence to indicate that myeloid cells are major contributors of immunopathology ${ }^{13-16}$. Whether post-COVID lung pathology is driven by persistence of this initial heightened inflammatory response or activation of alternative pathways that develop subsequent to recovery from the acute illness is unknown. Recent immunoprofiling studies have compared individuals with post-COVID19 lung parenchymal abnormalities to healthy controls and identified increases in airway CD8+ and CD4+ T lymphocytes with gene expression associated with myeloid cell inflammation ${ }^{17,18}$. These studies provide information about the immunological landscape 
after recovery from acute SARS-CoV-2 but lack an appropriate control group, namely individuals with resolution of lung changes post-COVID19 and so do not provide meaningful insight to distinguish the critical pathways dictating susceptibility to, or protection against, development of persistent interstitial lung changes.

A diverse range of aetiological factors and associated mechanisms are known to drive pathogenesis of other interstitial lung diseases ${ }^{19}$. The COVID19 pandemic offers a unique opportunity to study mechanisms of fibrogenesis in individuals with a defined common aetiology. Here, we utilise longitudinal systemic and airway sampling in a cohort with severe hospitalised COVID19 to compare individuals who have persistent interstitial lung changes to those with full radiographic resolution and elucidate the key immune mechanisms that drive this downstream complication. Using a multi-omic approach to gain broad unbiased insight, we identify that the development of post-COVID19 interstitial lung changes is associated with a pro-inflammatory neutrophil-associated immune signature detectable in the plasma and upper airways with a concomitant enriched anti-viral immune signature. Our findings provide fundamental functional insight into mechanisms driving pulmonary sequelae of COVID19 and provide a rational basis for development of future targeted therapeutic approaches to prevent longer term complications.

\section{Results}

To investigate the downstream immunological and clinical consequences following acute COVID19, we studied 46 subjects with severe COVID19. All subjects had clinical phenotyping, cross-sectional lung imaging, respiratory function testing and blood/nasal sampling at 3-6 months (mean 129 days) following discharge from hospital (visit 1 ). We additionally evaluated 18 subjects with mild (non-hospitalised) COVID19 and 17 healthy uninfected control subjects.

\section{Severe COVID19 is associated with an upregulated plasma immune signature following recovery from acute disease}

Acute COVID19 is characterised by mucosal and central hyperinflammation, a feature accentuated in individuals with more severe disease ${ }^{11,12,20}$ but whether immune activation persists following recovery from the acute illness is less well characterised. We initially examined the peripheral plasma proteome using the Olink proteomics platform to measure 184 unique proteins (Supplementary Table 1) and compared the immune signatures at 3-6 months post-discharge between subjects with severe COVID19 and those with mild COVID19 or healthy uninfected controls (Fig. 1A). The proteins measured were highly enriched for those involved in inflammation/immune processes. Principal component analysis (PCA) of plasma proteomes revealed differences between subjects with severe and mild COVID19 with separation of groups most evident along PC1 (Fig. 1B). 
To examine the effects of COVID19 severity on the plasma proteome at 3-6 months, we performed differential expression analysis. This identified 63 proteins with significantly altered concentrations ( $5 \%$ false discovery rate, $(F D R)$ ) with the majority $(n=59)$ upregulated and only 4 proteins downregulated (Fig. 1C\&D). The proteins that were most significantly differentially increased between severe and mild COVID19 patients (in terms of fold change) were CXCL5, HEXIM1, OSM and 4EBP1 (Fig. 1C). The downregulated proteins were CDSN, TPSAB1, CCL11 and PRDX1. Representative violin plots showing distributions of upregulated post-recovery plasma proteins in subjects with severe COVID19, mild COVID19 and healthy controls are shown in Fig. 1E. Pathway analysis of these 59 upregulated proteins using String-DB highlighted enrichment of gene ontology (GO) biological processes 'immune system process', 'immune response' and 'cytokine-mediated signalling pathway' (Fig. 1F). Collectively, these data indicated that severe COVID19 is associated with a persistent proinflammatory immune signature detectable in plasma at 3-6 months following recovery from the acute illness.

\section{Radiographic and lung function abnormalities occur in a sub-set of individuals post-recovery from severe COVID19 illness.}

Of the 46 subjects with severe COVID19, $n=26(56.2 \%)$ had interstitial lung changes on CT imaging at 3-6 months post-discharge with the remaining subjects $(n=20)$ showing no interstitial changes indicating full resolution of parenchymal changes (Extended Data Fig. 1A). Demographic and clinical characteristics of this cohort are shown in Supplementary Table 2. Persistent interstitial change on CT at 3-6 months postacute illness was associated with reduced pulmonary lung function (\% predicted Forced vital capacity and TLCO (transfer factor for carbon monoxide)( Extended Data Fig.1 B\&C), and increased symptom scores as measured by St Georges Respiratory Questionnaire (SGRQ) (Extended Data Fig. 1D).

\section{Post-COVID19 interstitial lung changes are characterised by an augmented pro-inflammatory neutrophil- associated plasma immune signature.}

We next compared plasma proteomic profiles (again using the Olink platform) at 3-6 months postdischarge between individuals with post-COVID19 interstitial lung changes versus those with radiographic resolution (Fig. $2 A$ ) to gain insight into the immune pathways/mediators that might mechanistically drive this complication. PCA of plasma proteomes revealed differences between subjects with post-COVID19 interstitial changes versus those with resolution, most evident along PC1 (Fig. 2B). Differential protein abundance analysis identified 30 proteins with significantly altered concentrations ( $5 \%$ FDR) with the majority $(n=26)$ upregulated and 4 proteins downregulated (Fig. 2C\&D). The proteins that were most significantly differentially increased between subjects with interstitial lung changes and those with resolution (in terms of fold change) were the pro-inflammatory cytokines IL17C, EN-RAGE, 
CCL20, CCL25 and TNF (Fig. 2D). Downregulated proteins were the same as those attenuated at 3-6 months in all severe versus mild COVID19 subjects (CDSN, TPSAB1, CCL11 and PRDX1, Fig. 1D).

Representative violin plots showing distributions of selected upregulated plasma proteins in subjects with interstitial lung changes and those with resolution are shown in Fig. 2E. Pathway analysis of the 26 upregulated proteins using String-DB highlighted enrichment of gene ontology (GO) biological processes including 'Neutrophil chemotaxis', 'humoral immune response' and 'defense response' (Fig. 2F). Given that individuals with post-COVID interstitial changes differed from those with resolution with regards to several factors (e.g. age, BMI, initial disease severity, see Supplementary Table 2) which may themselves be associated with greater initial inflammatory responses (and thus a persistence of inflammation) we conducted a multivariable analysis to determine whether upregulated proteins remained independently associated with the presence of interstitial lung changes after adjustment for confounding variables $(P<0.1$ on univariate analysis, see Supplementary Table 2$)$. This revealed that the neutrophil-associated cytokine IL-17C was the only plasma protein independently associated with the development of interstitial changes (OR 3.72; 95\% Cl $1.20-16.84 ; P=0.0403)$, further supporting a causal role for a neutrophil-related immune signature in driving this complication.

To gain greater insight into the mediators/pathways involved in development of post-COVID19 interstitial lung changes, we additionally investigated for correlations between measured immune proteins and severity of radiological changes or lung function impairment. In keeping with our finding that 'neutrophil chemotaxis' was the most significantly enriched immune pathway associated with interstitial lung changes (Fig. 2G), the neutrophil chemokines CXCL1 and CXCL8 negatively correlated with \% FVC. CXCL8 and the interferon-inducible lymphocyte chemokine CXCL9 also negatively correlated with \%TLCO. For radiological disease extent, positive correlations were observed for: CXCL8, the inflammasome associated pro-inflammatory cytokine IL-18 and its receptor IL-18R1 (Fig. 2H).

Collectively, these data reveal that post-COVID19 interstitial lung changes are associated with a persistent plasma neutrophil-associated pro-inflammatory immune signature.

\section{Post-COVID19 interstitial lung changes are associated with an upregulated pro-inflammatory and antiviral immune signature in the upper airways}

The nose is a major entry site for SARS-COV-2 and therefore represents the primary mucosal site of initial host immune responses ${ }^{21,22}$. Given our findings that post-COVID19 interstitial lung changes were characterised by an upregulated systemic pro-inflammatory immune signature, we next sought to determine whether similar changes occurred within the upper airways by evaluating nasal brushing samples taken at the same timepoint (3-6 months following hospital discharge). Of the 46 subjects with 
severe COVID19 included in the study, 26 individuals ( $n=16$ with interstitial lung changes and $n=10$ resolved) had samples with sufficient RNA concentration/quality to perform transcriptomic analysis (Fig. 3A), for which we used the Nanostring NCounter multiplex platform which profiles $>850$ immune response genes (Supplementary Table 3).

PCA of nasal transcriptomes at 3-6 months post-discharge revealed differences between subjects with post-COVID19 interstitial changes versus those with resolution, most evident along PC1 (Fig. 3B), although differences were less marked than for plasma (Fig. 2B) and there was considerable overlap in the spatial location of subjects with interstitial changes and those with resolution. Differential gene expression (DEG) analysis identified 53 genes (5\% FDR) upregulated in subjects with interstitial changes versus those with resolution. The genes most significantly upregulated were predominantly broadly related to antiviral defence (e.g. GBP5, IFIT2, OASL) or neutrophilic inflammation/inflammasome pathways (e.g. CXCL8, CXCR2, IL 1R2, NLRP3)(Fig. 3C\&D). Representative violin plots showing distributions of selected upregulated immune genes in subjects with interstitial lung changes and those with full resolution are shown in Fig. $\mathbf{3 E}$. This global activation of antiviral and pro-inflammatory immune pathways was further supported by pathway analysis which indicated enrichment of gene ontology (GO) biological processes related to 'Cytokine mediated signalling pathway' and 'Cellular response to type I interferon' (Fig. 3F) and by cellular deconvolution analysis which indicated an enrichment of neutrophils in subjects with post-COVID19 interstitial lung changes (Fig. 3G). These data fit with existing paradigms suggesting that increased SARS-CoV-2 viral load in the upper respiratory tract associated with severe disease drives a type I IFN signature which contributes to subsequent hyperinflammation and disease severity ${ }^{23}$ and that these changes persist in individuals who have post-COVID19 interstitial lung changes.

\section{Post-COVID19 interstitial lung changes are associated with increased systemic neutrophils, protease concentrations and markers of NET formation.}

Having identified an upregulated neutrophil-associated immune signature in individuals with postCOVID19 interstitial lung changes, we hypothesised that increased neutrophils in these individuals may drive fibrogenesis through formation of neutrophil extracellular traps (NET) and consequent release of extracellular matrix (ECM) degrading neutrophil proteases. We therefore evaluated total neutrophil counts, neutrophil protease concentrations and markers of NET formation in plasma taken from individuals with interstitial lung changes compared to those with resolution (Fig. 4A). Total neutrophil numbers were increased in individuals with post-COVID19 interstitial changes compared to those with radiographic resolution (Fig. 4B\&C). A similar increase was observed for plasma concentrations of the neutrophil protease myeloperoxidase concentrations which also correlated positively with radiological disease extent and negatively with lung function impairment (\% TLCO) (Fig. 4D). There were no differences in plasma neutrophil elastase or extracellular DNA concentrations between the two groups (Extended Data 
Fig. 2 \& 3). NETosis involves hypercituillination of nucleosomal histone proteins including $\mathrm{H} 3$ which can be quantified in plasma as a remnant of NET formation. Concentrations of H3R8 citrullinated nucleosomes were increased in individuals with post-COVID19 interstitial changes compared to those with radiographic resolution with a similar trend $(P=0.09)$ observed for the total amount of circulating intact nucleosomes as measured by the canonical H3.1 nucleosomes assay. (Fig. 4D\&E).

\section{Post-COVID19 interstitial lung changes are associated with an upregulated pro-inflammatory immune signature in PBMC phosphoproteomes}

To better understand the mechanisms underlying perturbation of inflammatory signalling in subjects with post-COVID19 interstitial changes, we performed phosphoproteomic analysis of peripheral blood mononuclear cells (PBMCs) taken from a subgroup of individuals at the same 3-6 month timepoint (Fig. 5A). PCA of the phosphoproteomics revealed that there were distinct differences between PBMCs isolated from subjects exhibiting interstitial changes compared to those with resolution (Fig. 5B). Despite this clustering, there was heterogeneity observed across PBMC samples derived from subjects with interstitial changes. Differential expression analysis of 5,934 phosphopeptides identified 30 phosphopeptides significantly enriched (Log Odds Ratio > 4.6 - 99\% probability of being differentially expressed) in subjects with interstitial changes compared to those with complete resolution, and 16 phosphopeptides in the resolved group (Fig. 5C). An examination of sites that were differentially regulated between the groups with consistent intragroup levels of phosphorylation identified a group of 15 phosphorylation markers (Fig. 5D). Four phosphorylation sites $\left(\mathrm{PHF}^{\mathrm{S} 899}, \mathrm{PTPN7}^{\mathrm{S} 44}, \mathrm{NPM} 1^{\mathrm{S} 137}, \mathrm{STK} 10^{\mathrm{T} 952}\right.$ ) exhibited increased phosphorylation in all resolved subjects compared to those with interstitial changes, notably STK10 is involved in regulating lymphocyte migration ${ }^{24}$. The 11 phosphorylation sites that were increased in all subjects with interstitial changes were located on proteins implicated in phagosome action (TBB1 ${ }^{\mathrm{S} 35}, \mathrm{TBB}^{\mathrm{T} 143}$, TUBA4A $\left.{ }^{\mathrm{S} 439}\right)$, cell migration $\left(\mathrm{CD} 244^{\mathrm{S} 336}, \mathrm{DMTN}^{\mathrm{S} 303}, \mathrm{MYO}^{\mathrm{S}} \mathrm{C}^{\mathrm{S} 408}\right.$ ), and actin cytoskeleton organisation (INF2 ${ }^{\mathrm{T} 1179}, \mathrm{DMTN}^{\mathrm{S} 303}$, and $\mathrm{MYO1C}^{\mathrm{S408}}$ ). Kinase substrate enrichment analysis (KSEA) was performed to identify kinase - kinase signalling axes that were enriched in either outcome post-COVID19. The enriched axes were then used to reconstruct kinase networks and ascertain which kinases were crucial for the maintaining network integrity (Fig. 5E, left panel). Analysis revealed the emergence of network dependencies around CDK2, PRKCl, IRAK1, MEK1 and JNK2 in PBMCs derived from subjects with interstitial changes. These kinases are downstream of receptors bound by proinflammatory cytokines (type-1 IFN and TNF) and regulate immune pathways required for both T- and B- cell activation (the predominant populations in PBMCs). The kinases responsible for maintaining network integrity in the resolved subjects were more diverse (PHKG2 - metabolic, YES1 - cell growth, RPS6KA2 - regulates proliferation, MINK1- cell adhesion, and numerous others), suggestive of cells that had returned to steady state (Figure 5E, right panel). There was also overlap with kinases observed in the interstitial group (e.g. ARAF) however, the underlying ARAF-signalling axes enriched in either group were different. 
Collectively, these phosphoproteomic data support our findings from the other complimentary -omic approaches whereby signalling in PBMCs from subjects with post-COVID19 interstitial lung changes are characterised by enrichment for proliferative (CDK2, MEK1) and immunoregulatory (IRAK1, JNK2, PRKCI) kinases downstream of receptors to pro-inflammatory cytokines.

\section{Resolution of interstitial lung changes and associated immune alterations by 12 months post COVID19}

We next questioned whether post-COVID lung interstitial changes and associated immune alterations resolve over time by assessing longitudinal progression at a second later timepoint (visit 2)(median 180 days duration between first and second sampling) (Fig. 6A). In the group with interstitial changes, visit 2 data were available for 19/26 individuals for \%TLCO and 20/26 for \%FVC. Analysis of change in \%TLCO from visit 1 to visit 2 in subjects with interstitial changes as a group indicated overall improvement (median(IQR) at visit 1: 48.0 (44.0-62.0)\% vs visit 2: 58.0 (52.5-68.4)\%, Fig. 6B). \% FVC similarly showed overall improvement in the overall group (median(IQR) at visit 1: 79.5(73.3-93.5)\% vs visit 2: 89.5 (82.193.9) \%, Fig. 5C). Despite this overall improvement, 16 out of 19 individuals still had TLCO $<75 \%$ at visit 2 , suggesting full normalisation had not yet occurred in majority of subjects (although with the caveat that pre-morbid lung function results are not available and some may have had pre-existing abnormalities). Repeat CT imaging was available in 16/26 individuals showed a trend towards overall improvement ( median(IQR) at visit 1: 62.5 (44.4-88.1\%) vs visit 260.0 (41.2-72.5\%), Fig. 6D). However, $15 / 16$ subjects still had disease extent of $20 \%$ or greater, indicating that full resolution had not yet occurred. Repeat St George's Respiratory Questionnaire scores were available in 17/26 individuals and also showed a similar trend towards improvement over time (Fig. 6E). This clinical resolution was mirrored by a similar longitudinal reduction of cytokines found to be increased at 3-6 months including TNF, IL-17C and CXCL8/IL-8 (Fig. 6F-H) and a similar attenuation in myeloperoxidase, H3.1 and H3R8 nucleosome concentrations (Fig. 6I-K). Collectively, these data indicate that, in the majority of subjects with post-COVID19 interstitial changes evident at 3-6 months, partial resolution of clinical and inflammatory abnormalities occurs, although full normalisation of lung function and radiographic changes has not yet been reached in many individuals.

\section{Discussion}

Numerous studies have assessed immune mechanisms dictating the early acute phases of SARS-CoV2 infection and these insights have led to development of efficacious therapies now incorporated into clinical practice ${ }^{25}$. However, characterisation of which mechanistic pathways drive longer term pulmonary sequelae of COVID19 remains a key question in the field. Using detailed clinical and radiological phenotyping in a cohort of individuals initially hospitalised for severe COVID19 with high invasive ventilation requirement, we define a subgroup of individuals who have persistent morphological 
and functional changes following recovery from their acute illness. Using multi-omic profiling of biological samples taken from the blood and airways of these individuals following hospital discharge, we elucidate that this complication is characterised by an upregulated pro-inflammatory/neutrophilassociated immune signature.

Acute severe COVID19 is characterised by an augmented pro-inflammatory profile detectable both systematically and in the respiratory tract ${ }^{11-13}$. This hyperinflammatory state is believed to drive disease severity and is amenable to therapeutic inhibition using broad (e.g. dexamethasone ${ }^{25}$ ) or more targeted (e.g. tofacitinib ${ }^{26}$ ) anti-inflammatory approaches. Whether this inflammatory signature persists following clinical recovery from the acute illness is less well studied. Our observations using plasma proteomics to compare individuals with severe hospitalised COVID19 to those with mild illness indicate that, even out to 3-6 months following hospital discharge, there is a detectable increased systemic inflammatory signal. This indicates that severe COVID19 induces immune activation persisting even after recovery from the acute illness. A recent study by Talla et al, characterising the longitudinal dynamics of mild COVID19, showed a significant decrease in pro-inflammatory cytokines with an increase in proteins enriched for angiogenesis and coagulation pathways during the recovery phase, indicating a transition from inflammatory to wound healing pathways ${ }^{27}$. Our data suggests that this transition may be delayed in severe COVID19 since pro-inflammatory signatures remained elevated compared to mild disease. This is supported by other studies in the literature that have shown increased systemic inflammation-associated markers in individuals recovering from acute severe COVID19 when compared to healthy control subjects ${ }^{17,28}$.

Having identified persistent immune activation following severe COVID19, we sought to determine whether components of this pro-inflammatory signature, or alternative pathways, are selectively upregulated in subjects with persistent morphologic abnormalities following COVID-19 compared to those showing radiographic resolution. Such comparisons have the potential to offer clearer discrimination of key mechanistic drivers than comparisons to healthy uninfected subjects, as has been the case in other studies ${ }^{17,18}$. The present study has identified a range of selectively increased plasma proteins in individuals with post-COVID interstitial lung changes predominantly consistent with a neutrophil-associated inflammatory signature. Pathway analysis revealed that 'neutrophil chemotaxis' was the most significantly enriched gene ontology pathway and a number of differentially expressed proteins identified were related to neutrophilic inflammation including the neutrophil chemokine IL-17C, the neutrophil-derived cytokine EN-RAGE and the pro-inflammatory cytokines TNF and CCL20. IL-17C has been shown to have a critical role in regulation of neutrophilic recruitment and inflammation alongside expression of CCL20 and EN-RAGE (S100A12) ${ }^{29-31}$. EN-RAGE has recently shown to be highly expressed in dysfunctional low-density neutrophils in individuals with severe COVID-19 ${ }^{32}$ and is a key regulator of 
host immune inflammation during infection including monocyte activation and recruitment via TLR4 ${ }^{33}$. The secretion of alarmins (e.g. S100A8/A9) have additionally been shown to mediate activation of dysregulated neutrophils in animal models and are released upon formation of neutrophil extracellular traps inducing neutrophil activation, adhesion and CD11b upregulation ${ }^{34,35}$ again reinforcing the likely importance of neutrophilic inflammation in the development of post-COVID-19 lung sequelae.

For further insight into functionally relevant proteins, we also assessed for correlations with radiographic and functional severity and found that neutrophil chemokines (e.g. CXCL1 and CXCL8) correlated most closely with severity of radiological abnormalities and lung function impairment. In keeping with this proteomic signature, total blood neutrophil counts measured at the same timepoint were also elevated in individuals with post-COVID interstitial changes. Taken together, these data indicate that an augmented neutrophil-associated immune signature detectable in the plasma characterises post-COVID interstitial lung changes. We additionally sought to determine whether similar changes occurred mucosally at the site of initial SARS-CoV-2 infection and identified a consistent transcriptomic signature in the nasal mucosa of individuals with interstitial lung changes, again at 3-6 months post-recovery. Differentially expressed genes included the neutrophil chemokine $C X C L 8$, the neutrophil-expressed receptor $C X C R 2$ and the inflammasome-related genes NLRP3 and ILR2. Furthermore, cellular deconvolution analysis revealed an enrichment of neutrophils (but not other cell types) in individuals with post-COVID interstitial lung changes compared to those showing resolution. The finding of upregulated systemic and mucosal neutrophil-associated inflammation is consistent with a persistence of this signature from acute disease which is exaggerated in those who have persistent abnormalities following COVID-19. Clinical studies in COVID19 have also reported elevated neutrophil counts in peripheral blood, especially in advanced latestage disease ${ }^{36,37}$ and higher systemic neutrophil counts, neutrophil chemokines levels and altered immunometabolism are associated with increased disease severity and/or adverse outcomes ${ }^{11,36,38}$. Neutrophil chemokine signatures are also upregulated during acute COVID19 in airway transcriptomic studies ${ }^{20}$. Therefore, ample evidence supports the role played by neutrophils in driving acute severe disease and our study extends this knowledge to indicate a previously unrecognised role also played in driving the longer term sequelae of COVID19.

Effector mechanisms of neutrophils include formation of NETs which comprise networks of fibres composed of DNA containing histones and enzymes including myeloperoxidase and neutrophil elastase ${ }^{39}$. NET formation may have protective and pathogenic roles; ample evidence indicates roles in initiation and propagation of inflammation in a range of clinical conditions including COVID19 ${ }^{40-42}$ and excessive NET formation can trigger a cascade leading to tissue destruction and end-organ damage ${ }^{43}$.

Mechanistically, neutrophils may therefore drive fibrogenesis through release of proteases (e.g. neutrophil elastase and matrix metalloproteins (MMPs)) that can degrade extracellular matrix (ECM) proteins such 
as types I-IV collagens ${ }^{44}$. Neutrophil elastase and selected MMPs (e.g. MMP-3 and -8 ) have been shown to have functional roles in driving bleomycin-induced fibrosis in mice ${ }^{45-48}$ Evidence of increased neutrophil activation and NET formation in lung tissue and BAL fluid from fibrotic ILD patients has also been shown, possibly driven by hypoxia and upregulation of HIF-1 $\mathrm{a}^{49}$. Furthermore, upregulation of genes related to pulmonary fibrosis, as well as those for neutrophil activation and NETosis have been demonstrated in acute fatal COVID-19 ${ }^{50}$, further emphasising the central role of these processes in acute severe disease. In keeping with the hypothesis that NETosis/release of proteases by neutrophils may also drive development of post-COVID interstitial lung changes, we found that patients with this complication had total neutrophilia, increased plasma myeloperoxidase concentrations and elevated total levels of intact nucleosomes (as measured by H3.1) as well as increased levels of citrullinated H3R8 nucleosomes. These citrullinated nucleosomes are a major component of NETs as the NETosis process involves nucleosomal histone proteins $\mathrm{H} 3, \mathrm{H} 2 \mathrm{~A}$ and $\mathrm{H} 4$ being hypercitrullinated by the enzyme PAD4. However, PAD4 is dispensible for NETosis and non-citrullinated NETs are well recognised but likely not as inflammatory as their citrullinated counterparts ${ }^{51,52}$. Accordingly, extracellular histones are elevated in airway fluid of patients with ARDS ${ }^{53}$ and inhibition of NETosis reduces lung injury and increases survival in animal ARDS models 54,55 .

Although we were unable to perform functional experiments to confirm a direct causal role of neutrophils/NETS in driving development post-COVID interstitial lung changes in the current study, our finding that systemic neutrophil chemokine and myeloperoxidase levels and nasal IL $17 D$ significantly correlated with severity of radiographic and lung function impairment in individuals with this complication provides support for the hypothesis. Furthermore, after correction for other confounding variables (age, disease severity etc.), plasma concentrations of the neutrophil-regulating cytokine IL-17C remained independently associated with post-COVID19 interstitial lung changes. IL-17C was also recently shown to be associated with disease progression in fibrosing interstial lung disease associated with connective tissue disease, chronic hypersensitivity pneumonitis or unclassifiable origin ${ }^{56}$ which further supports a broader role of this pathway in fibrogenesis. Notably, neutrophil priming and NET formation are poorly responsive to corticosteroids ${ }^{42}$ which are now widely used for severe COVID19, emphasising that alternative approaches are needed. Future functional studies in mouse models of SARS-CoV2 (including a recently described humanised system that recapitulates disease features including pulmonary fibrosis ${ }^{57}$ ) and/or longitudinal analysis of ongoing clinical trials examining effects of neutrophil targeting therapies such as elastase inhibitors (e.g. NCT04817332) upon development of postCOVID interstitial changes will be required to provide formal confirmation of causal roles. However, it is interesting to note that there were no differences in total neutrophil elastase levels between the interstitial changes and resolved groups in our study which does not lend support to the use of neutrophil elastase inhibitors after recovery from acute illness. 
In addition to an upregulated neutrophil-associated immune signature detected in the nose, we also identified differentially expressed genes related to augmented antiviral defence pathways. This was supported by our finding that the interferon-inducible cytokine CXCL9 was increased within the plasma proteome of individuals with post-COVID19 interstitial changes and negatively correlated with gas transfer lung function measurements. Original conceptual thinking related to SARS-COV-2 infection was that severe disease is associated with impaired type I IFN responses but increased NFkB driven proinflammatory responses ${ }^{58}$. However, increasing evidence indicates greater complexity with airwayfocussed studies reporting increased interferon-stimulated gene (ISG) expression occurring concomitantly with pro-inflammatory and chemokines genes ${ }^{20}$. Single cell sequencing studies in patients with acute COVID19 have revealed hyper-inflammatory immune signatures (TNF/IL1 $\beta$ ) across all types of immune cells and co-occurring with type I IFN in classical monocytes in severe but not mild disease ${ }^{59}$ Animal studies have indicated pro-inflammatory roles for type I IFN during SARS-CoV-2 infection ${ }^{60}$ with excessive or prolonged IFN production post viral infection impairing lung epithelial regeneration ${ }^{61}$. The use of JAK inhibitors which interfere with type-I IFN signalling can limit proinflammatory cytokine release ${ }^{62,63}$. Our data indicates that post-COVID interstitial changes are associated with a persistence of airway type I IFN signature and we interpret that this relates to an initial heightened antiviral response due to increased severity of initial infection (as indicated by greater disease severity e.g. requirement for invasive ventilation) observed in the post-COVID interstitial changes group. Our data therefore support a potential mechanism where more severe initial SARS-CoV-2 infection induces a greater type I IFN response which drives subsequent proinflammatory neutrophilic responses with neutrophil NET formation and protease release potentially leading to extracellular matrix degradation and development of interstitial lung changes. NETs are known to signal intracellularly via the cGAS-STING pathway to release type I IFN ${ }^{64}$. This pathway has been shown to be activated within the lung tissue of severe acute COVID-19 patients and further reinforces the importance of NETs in the pathogenesis of severe disease ${ }^{65}$.

Alternatively, some recent studies report expansion of neutrophil sub-populations characterised by type I interferon signalling in severe COVID 19 66,67 with demonstrated modulation by corticosteroids ${ }^{66}$, but the relative contribution of these cells to the overall antiviral response in comparison to the other major producers of type I interferon (e.g. epithelium, dendritic cells) remains unclear. It also remains unknown at which point therapies would need to be administered to target these pathways and interrupt this process, although some authors have speculated that this should occur during the early phases of disease ${ }^{10}$. Whether acute COVID19 therapies that would suppress type I IFN signalling (e.g. JAK inhibitors) or neutrophil-mediated inflammation (e.g. neutrophil elastase inhibitors, extracorporeal removal of NETs via selective therapeutic apheresis) can effectively reduce the likelihood of long term pulmonary sequelae requires further study. 
To gain more detailed mechanistic insight into the key alterations in inflammatory signalling pathways, we utilised phosphoproteomic evaluation of PBMCs which uncovered a range of kinases enriched in individuals with post-COVID19 interstitial lung changes. Consistent with our proteomic and transcriptomic data, a number of these enriched kinases play key roles in pro-inflammatory/neutrophilic pathways and antiviral immunity. These include CDK2 (a canonical cell cycle regulator with roles in neutrophil migration ${ }^{68}$ ), IRAK1 (a key component of inflammasome/TLR signalling and type I interferon pathways ${ }^{69,70}$ and JNK2 which has roles in proinflammatory cytokine responses and neutrophil effector functions ${ }^{71}$. Moreover, phosphorylation sites increased in subjects with interstitial changes also included proteins implicated in actin cytoskeleton organisation. Actin cytoskeleton breakdown is an early feature in the sequence of cellular events occurring in NETosis and precedes plasma/nuclear permealisation and chromatin decondensation ${ }^{72,73}$. This is consistent with our finding that markers of NETosis are increased in individuals with post-COVID19 interstitial lung changes and further supports a potential mechanistic role for neutrophil associated inflammatory pathways in driving this complication. Importantly, a range of licenced or late-stage developmental therapies are available that target these kinases/pathways ${ }^{74-76}$ and future studies should be directed at studying the utility of these agents in ameliorating post-COVID interstitial lung disease.

Sequential evaluation and sampling of our cohort at a second follow-up visit $~ 6$ months following the initial 3-6 month assessment indicated that abnormalities in lung function, radiographic changes and symptom scores show evidence of significant improvement with time. However, abnormalities in lung function (particularly TLCO), radiological changes and symptom scores have not fully normalised at this timepoint (although it should be noted that we do not have information about pre-morbid levels of these variables from subjects included in our study). Whether full normalisation of these parameters occurs by a later timepoint (e.g. 18 or 24 months) requires future study and it is unclear whether some individuals will suffer from long-term chronic post-COVID interstitial lung fibrosis. The true long-term burden of this complication remains unknown and the results of ongoing large-scale cohort studies are awaited ${ }^{77}$.

In conclusion, our study uncovers new mechanistic insight into the immune pathways driving postCOVID19 interstitial changes. The longer term implications of whether chronic changes occur in a subset of these individuals and the potential of targeting neutrophilic inflammation, either in the acute or recovery phase of COVID19, to limit long-term disease sequelae requires further study.

\section{References}

1. Docherty, A.B., et al. Features of 20133 UK patients in hospital with covid-19 using the ISARIC WHO Clinical Characterisation Protocol: prospective observational cohort study. BMJ 369, m1985 (2020). 
2. Evans, R.A., et al. Physical, cognitive, and mental health impacts of COVID-19 after hospitalisation (PHOSP-COVID): a UK multicentre, prospective cohort study. The Lancet. Respiratory medicine 9, 12751287 (2021).

3. Augustin, M., et al. Post-COVID syndrome in non-hospitalised patients with COVID-19: a longitudinal prospective cohort study. Lancet Reg Health Eur 6, 100122 (2021).

4. Nalbandian, A., et al. Post-acute COVID-19 syndrome. Nature medicine 27, 601-615 (2021).

5. Han, X., et al. Six-month Follow-up Chest CT Findings after Severe COVID-19 Pneumonia. Radiology 299, E177-E186 (2021).

6. Guler, S.A., et al. Pulmonary function and radiological features 4 months after COVID-19: first results from the national prospective observational Swiss COVID-19 lung study. The European respiratory journal : official journal of the European Society for Clinical Respiratory Physiology 57(2021).

7. $\mathrm{Xu}, \mathrm{Y} . \mathrm{H} .$, et al. Clinical and computed tomographic imaging features of novel coronavirus pneumonia caused by SARS-CoV-2. The Journal of infection $80,394-400$ (2020).

8. Mo, X., et al. Abnormal pulmonary function in COVID-19 patients at time of hospital discharge. The European respiratory journal : official journal of the European Society for Clinical Respiratory Physiology 55(2020).

9. Li, X., et al. Pulmonary fibrosis and its related factors in discharged patients with new corona virus pneumonia: a cohort study. Respiratory research 22, 203 (2021).

10. George, P.M., Wells, A.U. \& Jenkins, R.G. Pulmonary fibrosis and COVID-19: the potential role for antifibrotic therapy. The Lancet. Respiratory medicine 8, 807-815 (2020).

11. Thwaites, R.S., et al. Inflammatory profiles across the spectrum of disease reveal a distinct role for GM-CSF in severe COVID-19. Sci Immunol 6(2021).

12. Melms, J.C., et al. A molecular single-cell lung atlas of lethal COVID-19. Nature 595, 114-119 (2021).

13. Rendeiro, A.F., et al. The spatio-temporal landscape of lung pathology in SARS-CoV-2 infection. medRxiv (2020).

14. Mann, E.R., et al. Longitudinal immune profiling reveals key myeloid signatures associated with COVID-19. Sci Immunol 5(2020).

15. Grant, R.A., et al. Circuits between infected macrophages and T cells in SARS-CoV-2 pneumonia. Nature 590, 635-641 (2021). 
16. Dorward, D.A., et al. Tissue-Specific Immunopathology in Fatal COVID-19. American journal of respiratory and critical care medicine 203, 192-201 (2021).

17. Vijayakumar, B., Boustani, K., Ogger, P.P. \& et al. Immuno-proteomic profiling reveals abundant airway CD8 T cells and ongoing epithelial injury in prolonged post-COVID19 respiratory disease. medRxiV doi: https://doi.org/10.1101/2021.08.10.21261834(2021).

18. Cheon, I.S., et al. Immune signatures underlying post-acute COVID-19 lung sequelae. Sci Immunol 6, eabk1741 (2021).

19. Selman, M. \& Pardo, A. When things go wrong: exploring possible mechanisms driving the progressive fibrosis phenotype in interstitial lung diseases. The European respiratory journal : official journal of the European Society for Clinical Respiratory Physiology 58(2021).

20. Zhou, Z., et al. Heightened Innate Immune Responses in the Respiratory Tract of COVID-19 Patients. Cell Host Microbe 27, 883-890 e882 (2020).

21. Sposito, B., et al. The interferon landscape along the respiratory tract impacts the severity of COVID-19. Cell 184, 4953-4968 e4916 (2021).

22. Hatton, C.F., et al. Delayed induction of type I and III interferons mediates nasal epithelial cell permissiveness to SARS-CoV-2. Nat Commun 12, 7092 (2021).

23. Park, A. \& Iwasaki, A. Type I and Type III Interferons - Induction, Signaling, Evasion, and Application to Combat COVID-19. Cell Host Microbe 27, 870-878 (2020).

24. Zhang, L., et al. STK10 knockout inhibits cell migration and promotes cell proliferation via modulating the activity of ERM and p38 MAPK in prostate cancer cells. Exp Ther Med 22, 851 (2021).

25. Group, R.C., et al. Dexamethasone in Hospitalized Patients with Covid-19. The New England journal of medicine $384,693-704$ (2021).

26. Guimaraes, P.O., et al. Tofacitinib in Patients Hospitalized with Covid-19 Pneumonia. The New England journal of medicine 385, 406-415 (2021).

27. Talla, A., et al. Longitudinal immune dynamics of mild COVID-19 define signatures of recovery and persistence. bioRxiv (2021).

28. Ong, S.W.X., et al. Persistent Symptoms and Association With Inflammatory Cytokine Signatures in Recovered Coronavirus Disease 2019 Patients. Open Forum Infect Dis 8, ofab156 (2021).

29. Jamieson, K.C., et al. Rhinovirus and Bacteria Synergistically Induce IL-17C Release from Human Airway Epithelial Cells To Promote Neutrophil Recruitment. J Immunol 202, 160-170 (2019). 
30. Nies, J.F. \& Panzer, U. IL-17C/IL-17RE: Emergence of a Unique Axis in TH17 Biology. Front Immunol $11,341(2020)$.

31. Kusagaya, H., et al. Toll-like receptor-mediated airway IL-17C enhances epithelial host defense in an autocrine/paracrine manner. American journal of respiratory cell and molecular biology 50, 30-39 (2014).

32. Schulte-Schrepping, J., et al. Severe COVID-19 Is Marked by a Dysregulated Myeloid Cell Compartment. Cel/ 182, 1419-1440 e1423 (2020).

33. Foell, D., et al. Proinflammatory S100A12 can activate human monocytes via Toll-like receptor 4 . American journal of respiratory and critical care medicine 187, 1324-1334 (2013).

34. Guo, Q., et al. Induction of alarmin S100A8/A9 mediates activation of aberrant neutrophils in the pathogenesis of COVID-19. Cell Host Microbe 29, 222-235 e224 (2021).

35. Sprenkeler, E.G.G., Zandstra, J., van Kleef, N.D. \& et al. S100A8/A9 is a marker of the release of neutrophil extracellular traps and induces neutrophil activation. Cells 11 (2), 236. (2022).

36. Huang, D., et al. Clinical features of severe patients infected with 2019 novel coronavirus: a systematic review and meta-analysis. Ann Trans/ Med 8, 576 (2020).

37. Wang, D., et al. Clinical Characteristics of 138 Hospitalized Patients With 2019 Novel CoronavirusInfected Pneumonia in Wuhan, China. JAMA : the journal of the American Medical Association 323, 10611069 (2020).

38. McElvaney, O.J., et al. Characterization of the Inflammatory Response to Severe COVID-19 IIIness. American journal of respiratory and critical care medicine 202, 812-821 (2020).

39. Brinkmann, V., et al. Neutrophil extracellular traps kill bacteria. Science 303, 1532-1535 (2004).

40. Zuo, Y., et al. Neutrophil extracellular traps in COVID-19. JCl Insight 5(2020).

41. Veras, F.P., et al. SARS-CoV-2-triggered neutrophil extracellular traps mediate COVID-19 pathology. $J$ Exp Med 217(2020).

42. Panda, R., et al. A functionally distinct neutrophil landscape in severe COVID-19 reveals opportunities for adjunctive therapies. JCI Insight (2021).

43. Barnes, B.J., et al. Targeting potential drivers of COVID-19: Neutrophil extracellular traps. J Exp Med 217(2020).

44. Mincham, K.T., Bruno, N., Singanayagam, A. \& Snelgrove, R.J. Our evolving view of neutrophils in defining the pathology of chronic lung disease. Immunology 164, 701-721 (2021). 
45. Chua, F., et al. Mice lacking neutrophil elastase are resistant to bleomycin-induced pulmonary fibrosis. The American journal of pathology 170, 65-74 (2007).

46. Takemasa, A., Ishii, Y. \& Fukuda, T. A neutrophil elastase inhibitor prevents bleomycin-induced pulmonary fibrosis in mice. The European respiratory journal : official journal of the European Society for Clinical Respiratory Physiology 40, 1475-1482 (2012).

47. Yamashita, C.M., et al. Matrix metalloproteinase 3 is a mediator of pulmonary fibrosis. The American journal of pathology 179, 1733-1745 (2011).

48. Garcia-Prieto, E., et al. Resistance to bleomycin-induced lung fibrosis in MMP-8 deficient mice is mediated by interleukin-10. PloS one 5, e13242 (2010).

49. Khawaja, A.A., et al. Identification of a Novel HIF-1 alpha-alphaMbeta2 Integrin-NET Axis in Fibrotic Interstitial Lung Disease. Front Immunol 11, 2190 (2020).

50. Wu, M., et al. Transcriptional and proteomic insights into the host response in fatal COVID-19 cases. Proceedings of the National Academy of Sciences of the United States of America 117, 2833628343 (2020).

51. Tsourouktsoglou, T.D., et al. Histones, DNA, and Citrullination Promote Neutrophil Extracellular Trap Inflammation by Regulating the Localization and Activation of TLR4. Cell Rep 31, 107602 (2020).

52. Huckriede, J., et al. Histone H3 Cleavage in Severe COVID-19 ICU Patients. Front Cell Infect Microbiol 11, 694186 (2021).

53. Lv, X., et al. Extracellular histones are clinically relevant mediators in the pathogenesis of acute respiratory distress syndrome. Respiratory research 18, 165 (2017).

54. Lefrancais, E., Mallavia, B., Zhuo, H., Calfee, C.S. \& Looney, M.R. Maladaptive role of neutrophil extracellular traps in pathogen-induced lung injury. JCI Insight 3(2018).

55. Liu, S., et al. Neutrophil extracellular traps are indirectly triggered by lipopolysaccharide and contribute to acute lung injury. Scientific reports 6, 37252 (2016).

56. Bowman, W.S., et al. Proteomic biomarkers of progressive fibrosing interstitial lung disease: a multicentre cohort analysis. The Lancet. Respiratory medicine [Online](2022).

57. Sefik, E., et al. A humanized mouse model of chronic COVID-19. Nat Biotechnol (2021).

58. Hadjadj, J., et al. Impaired type I interferon activity and inflammatory responses in severe COVID19 patients. Science $369,718-724$ (2020).

59. Lee, J.S., et al. Immunophenotyping of COVID-19 and influenza highlights the role of type I interferons in development of severe COVID-19. Sci Immuno/ 5(2020). 
60. Israelow, B., et al. Mouse model of SARS-CoV-2 reveals inflammatory role of type I interferon signaling. J Exp Med 217(2020).

61. Major, J., et al. Type I and III interferons disrupt lung epithelial repair during recovery from viral infection. Science 369, 712-717 (2020).

62. Sandborn, W.J., et al. Tofacitinib as Induction and Maintenance Therapy for Ulcerative Colitis. The New England journal of medicine 376, 1723-1736 (2017).

63. Gadina, M., et al. Translational and clinical advances in JAK-STAT biology: The present and future of jakinibs. Journal of leukocyte biology 104, 499-514 (2018).

64. Apel, F., et al. The cytosolic DNA sensor cGAS recognizes neutrophil extracellular traps. Sci Signal 14(2021).

65. Di Domizio, J., et al. The cGAS-STING pathway drives type I IFN immunopathology in COVID-19. Nature (2022).

66. Sinha, S., et al. Dexamethasone modulates immature neutrophils and interferon programming in severe COVID-19. Nature medicine (2021).

67. Reyes, L., et al. ---A type I IFN, prothrombotic hyperinflammatory neutrophil signature is distinct for COVID-19 ARDS. Wellcome Open Res 6, 38 (2021).

68. Hsu, A.Y., et al. Phenotypical microRNA screen reveals a noncanonical role of CDK2 in regulating neutrophil migration. Proceedings of the National Academy of Sciences of the United States of America $116,18561-18570$ (2019).

69. Heiseke, A.F., et al. IRAK1 Drives Intestinal Inflammation by Promoting the Generation of Effector Th Cells with Optimal Gut-Homing Capacity. J Immuno/ 195, 5787-5794 (2015).

70. Dumbrepatil, A.B., et al. Viperin interacts with the kinase IRAK1 and the E3 ubiquitin ligase TRAF6, coupling innate immune signaling to antiviral ribonucleotide synthesis. The Journal of biological chemistry 294, 6888-6898 (2019).

71. Sukhumavasi, W., Egan, C.E. \& Denkers, E.Y. Mouse neutrophils require JNK2 MAPK for Toxoplasma gondii-induced IL-12p40 and CCL2/MCP-1 release. J Immuno/ 179, 3570-3577 (2007).

72. Sprenkeler, E.G.G., Tool, A.T.J., Henriet, S., van Bruggen, R. \& Kuijpers, T.W. Formation of neutrophil extracellular traps requires actin cytoskeleton rearrangements. Blood(2022).

73. Thiam, H.R., et al. NETosis proceeds by cytoskeleton and endomembrane disassembly and PAD4mediated chromatin decondensation and nuclear envelope rupture. Proceedings of the National Academy of Sciences of the United States of America 117, 7326-7337 (2020). 
74. Singer, J.W., et al. Inhibition of interleukin-1 receptor-associated kinase 1 (IRAK1) as a therapeutic strategy. Oncotarget 9, 33416-33439 (2018).

75. Leitch, A.E., Haslett, C. \& Rossi, A.G. Cyclin-dependent kinase inhibitor drugs as potential novel anti-inflammatory and pro-resolution agents. Br J Pharmacol 158, 1004-1016 (2009).

76. Wu, Q., et al. Selective inhibitors for JNK signalling: a potential targeted therapy in cancer. $J$ Enzyme Inhib Med Chem 35, 574-583 (2020).

77. Wild, J.M., et al. Understanding the burden of interstitial lung disease post-COVID-19: the UK Interstitial Lung Disease-Long COVID Study (UKILD-Long COVID). BMJ Open Respir Res 8(2021).

78. Huang, C., et al. 6-month consequences of COVID-19 in patients discharged from hospital: a cohort study. Lancet 397, 220-232 (2021).

79. Khorsandi, S.E., et al. Computational Analysis of Cholangiocarcinoma Phosphoproteomes Identifies Patient-Specific Drug Targets. Cancer Res 81, 5765-5776 (2021).

80. Perkins, D.N., Pappin, D.J., Creasy, D.M. \& Cottrell, J.S. Probability-based protein identification by searching sequence databases using mass spectrometry data. Electrophoresis 20, 3551-3567 (1999).

81. Ritchie, M.E., et al. limma powers differential expression analyses for RNA-sequencing and microarray studies. Nucleic Acids Res 43, e47 (2015).

82. Hijazi, M., Smith, R., Rajeeve, V., Bessant, C. \& Cutillas, P.R. Reconstructing kinase network topologies from phosphoproteomics data reveals cancer-associated rewiring. Nat Biotechno/ 38, 493-502 (2020).

83. Casado, P., et al. Kinase-substrate enrichment analysis provides insights into the heterogeneity of signaling pathway activation in leukemia cells. Sci Signal 6, rs6 (2013).

84. Cavalier, E., et al. Circulating Nucleosomes as Potential Markers to Monitor COVID-19 Disease Progression. Front Mol Biosci 8, 600881 (2021).

\section{Materials And Methods}

\section{Study design and participants}

This study prospectively recruited individuals with COVID 19 confirmed by positive polymerase chain reaction (PCR) from 01/03/2020 to 01/11/2021. All participants provided informed written consent.

Clinical demographics during acute illness including disease severity, medications, risk factors and comorbidities were recorded prospectively at the time of study entry. COVID19 disease severity was characterised by the highest seven-category scale during the hospital stay (termed the severity), which 
consisted of the following categories: 1- not admitted to hospital with resumption of normal activities; 2 not admitted to hospital, but unable to resume normal activities; 3 - admitted to hospital but not requiring supplemental oxygen; 4 -admitted to hospital but requiring supplemental oxygen; 5 -admitted to hospital requiring high-flow nasal cannula (HFNC), non-invasive mechanical ventilation (NIV), or both; 6- admitted to hospital requiring extracorporeal membrane oxygenation, invasive mechanical ventilation (IMV), or both; and 7 -death ${ }^{78}$. Participants with scores 5-7 were categorised as 'severe' disease with participants with scores 1-3 deemed 'mild'.

All participants were sampled following hospital discharge at 3-6 months (visit 1) and those with severe disease were invited to attend for further clinical assessment and sampling at $\sim 6$ months following this (visit 2). Healthy individuals were recruited as disease controls for the study and sampled once. At baseline and follow-up timepoints, venous blood sampling and nasal brushings were performed alongside a validated quality of life questionnaire (St. George Respiratory Questionnaire). Participants with severe disease had computed tomography (CT) chest imaging and pulmonary function tests as part of standard clinical post-COVID19 follow-up. Data were managed using REDCap electronic data capture tools in order to minimise missing inputs and allow for real-time data validation and quality control.

\section{Radiological assessment}

CT scans were assessed independently by two experienced thoracic radiologists blinded to clinical outcome data. Scans were initially categorised as (i) showing resolution of the parenchymal lung changes defined as $<5 \%$ abnormality present or (ii) having persistent parenchymal lung abnormalities. In patients with persistent abnormalities, the extent of abnormal lung to the nearest $5 \%$ was first recorded by each radiologist. Following this, the relative proportions (as a percentage) of abnormal lung comprising ground-glass opacification, parenchymal, bands, consolidation and reticulation (not exceeding 100\%) were quantified. Where present, the severity of traction bronchiectasis/bronchiolectasis was scored semiquantitavely $(0=$ no traction bronchiectasis/bronchiecatasis visible in abnormal lung; $1=$ mild; $2=$ moderate and 3-severe). The number of lobes in which traction bronchiectasis/bronchiecatasis were present was recorded. Finally the presence of volume loss in any lobe ( $0=$ no lobar volume loss; $1=$ mild; $2=$ moderate and $3=$ severe) was recorded Discrepancies of over $\geq 20 \%$ in extent for any CT pattern or more than 1 grade difference (for semi-quantitatively graded patterns) were resolved by consensus review.

\section{Sample collection and PBMC isolation}

Venous blood was collected in lithium heparin and EDTA blood tubes and transported at room temperature. Plasma was isolated from EDTA tubes following centrifugation at $1200 \mathrm{~g}$ for 10 minutes, with plasma aspirated and stored at $-80^{\circ} \mathrm{C}$. Venous blood from lithium heparin tubes was diluted 1:1 with phosphate buffered saline, layered over histopaque (Sigma Aldrich) and peripheral blood mononuclear cells (PBMCs) isolated following density gradient centrifugation. PBMCs were resuspended in 10\%DMSO and fetal calf serum and stored in liquid nitrogen. Nasal epithelial cells were collected by gently brushing 
the inferior turbinate of the nostril with a cut bronchoscopic cytology brush (Conmed) and placing the brush into a microfuge tube containing $1 \mathrm{~mL}$ DNA/RNA shield reagent (Zymoresearch) followed by storage at $-80^{\circ} \mathrm{C}$.

\section{Proteomic assays}

Plasma proteomic measurements were performed using Olink proximity extension immunoassays (https://www.olink.com/products/). Two 92-protein multiplex Olink panels were run ('inflammation' and 'immune response') resulting in 184 measurements per sample. Since a small number of proteins (3) were measured on more than one panel, we therefore measured a total of 181 unique proteins. The Olink assays were run using 88 samples/plate. All plates were run in a single batch. Plate layouts were designed to avoid confounding of potential plate effects with biological or clinical variables of interest ensuring case/control balance across plates with random selection of samples from each category and random ordering of allocation to wells. Specifically, a fixed proportion of each plate was designated for control samples. The data was normalised using standard Olink workflows to produce relative protein abundance on a log2 scale ('NPX'). Quality assessment was performed by (1) examination of Olink internal controls and (2) inspection of boxplots, relative log expression plots (Gandolfo and Speed, 2018), and PCA.

\section{Nanostring nCounter analysis}

Total RNA was extracted from nasal brush samples using a Qiagen RNAeasy kit following manufacturers instruction. Nanostring profiling of host response was performed using the nCounter Human Host

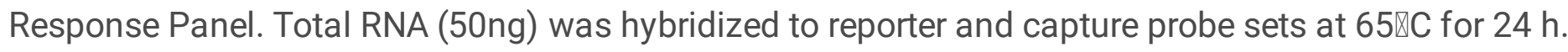
Probeset-target RNA complexes were purified and immobilized on nCounter ${ }^{\circledR}$ Cartridges using an nCounter® Prep Station ( $3 \mathrm{~h}$ and 20 min total time). Data collection was carried out in the nCounter® Digital Analyzer; at the highest standard data resolution, 555 Fields of View (FOV) were collected ( $6 \mathrm{~h}$ total time) per flow cell using a microscope objective and a CCD camera yielding data of all the target molecule counts. Raw data were pre-processed using both nSolver 2.0 software (Nanostring Technologies) and the Rosalind packages. Pre-processing sequentially corrects for three factors: technical variation, background and sample content. First, using a set of exogenous positive control RNAs present in each sample, technical variation is accounted for by adjusting the counts in each sample to the geometric mean of counts for positive control counts in all samples. Subsequently, a background correction is performed by subtracting the maximum count value of the negative control probes in a sample from each probe output within the sample. Transcripts counts that are negative after background correction are set to 1. Each sample is then normalized for RNA content by adjusting the counts to the geometric mean of 15 housekeeping genes. Finally, the data were log 2 transformed. Network data were designed by association matrix and the graph file was created using Gephi. Nodes 
were aligned by Force Atlas algorithm and the centrality was calculated by the betweenness. Downstream statistical analyses were performed using Graphpad Prism version 9.0

\section{Phosphoproteomic assays}

$3 \times 10^{6}$ PBMCs were washed with PBS and lysed with a buffer containing $8 \mathrm{M}$ urea in $20 \mathrm{mM}$ HEPES (pH 8) supplemented with $1 \mathrm{mM} \mathrm{Na}_{3} \mathrm{VO}_{4}, 1 \mathrm{mM} \mathrm{NaF}, 1 \mathrm{mM} \mathrm{Na} \beta$-glycerol phosphate and $2.5 \mathrm{mM} \mathrm{Na}_{2} \mathrm{H}_{2} \mathrm{P}_{2} \mathrm{O}_{7}$. The resultant lysates were then processed as described ${ }^{79}$. Briefly, samples were sonicated; normalised to $110 \mu \mathrm{g}$ of protein through the use of a Pierce ${ }^{\mathrm{TM}}$ bicinchoninic acid (BCA) assay, following which samples were subjected to tryptic digestion before desalting and phosphoenrichment.

Enriched phosphopeptides were loaded onto an LC-MS/MS system consisting of a Dionex UltiMate 3000 RSLC directly coupled to an Orbitrap Q-Exactive Plus mass spectrometer (Thermo Fisher Scientific). The LC method comprised mobile phases A ( $3 \%$ ACN: $0.1 \%$ FA) and B (100\% ACN; $0.1 \%$ FA). Peptides were trapped on a $\mu$-pre-column (Thermo Scientific) at $10 \mu \mathrm{L} / \mathrm{min}$ flow rate and separated on a $50 \mathrm{~cm}$ EASYSpray $^{\text {TM }}$ (Thermo Scientific) LC Column with a gradient of 3-23\% B (across 90 minutes) and a flow rate of $0.3 \mu \mathrm{L} /$ minute. The mass spectrometer operated with a 2.1 second duty cycle. MS1 survey spectra $(\mathrm{m} / \mathrm{z}$ 375-1,500) were acquired with a resolution of 70,000 and followed a data-dependent acquisition method in which the 10 most intense ions were selected for fragmentation, subsequent fragments (MS2; scanning $200-2,000 \mathrm{~m} / \mathrm{z}$ ) acquired with a resolution of 17,500. Dynamic exclusion set to 30 seconds.

Peptide identification steps systematically searched LC-MS/MS data against the SwissProt human database using Mascot 2.5 (Matrix Science) ${ }^{80}$. Allowable peptide modifications included phospho-Ser (S), phospho-Thr (T), phospho-Tyr (Y), oxidised Met (M), and pyro-Glu (Q). Phosphopeptide intensities were quantified in each sample through the use of in-house PiQuant software. Missing values were imputed using a multiple imputation strategy. In brief, this involves imputation of missing-completely-atrandom (MCAR) values with a maximum likelihood estimator (MLE) approach, following by imputation of missing-not-at-random (MNAR) values with an imputation under a Gaussian complete data assumption (IGCDA) approach. Data were then scale-normalised and differential expression analysis performed through the fitting of an empirical Bayes linear model ${ }^{81}$. The prior probability for differential expression was set at $1 \%$. A posterior probability of differential expression $>0.99$ (translating to a log-odds ratio $>$ 4.599) was considered strong evidence of differential expression. Where provided in the differential expression analyses, PP values were adjusted for multiple testing through the use of the BenjaminiYekutieli procedure. Kinase activities from the phosphoproteomics data calculated using Kinase Substrate Enrichment Analysis (KSEA) as described previously ${ }^{82,83}$. 


\section{ELISA assays}

Cytokine protein concentrations in plasma (TNF, CXCL8/IL-8, myeloperoxidase and neutrophil elastase) were assayed using commercial 'duoset' enzyme-linked immunosorbent assay kits (R\&D Systems, Abingdon, UK), according to Manufacturer's instructions. Plasma concentrations of cell-free DNA were quantified using the Quant-iT PicoGreen dsDNA Assay Kit (Invitrogen, Thermo Fisher Scientific) according to the manufacturer's instructions.

Levels of circulating H3.1 nucleosomes and H3R8cit nucleosomes were measured using Nu.Q ${ }^{\text {TM }}$ ELISA assays (Belgian Volition SRL, Isnes, Belgium) according to the manufacturer's instructions, as previously described $^{84}$. Data were analysed using Prism version 9 software (GraphPad, La Jolla CA, US).

\section{Declarations}

Ethics: The study was approved by the South Central Oxford A Research Ethics Committee (Ref: 20/SC/0208). All participants provided informed written consent.

Conflicts of interest: PMG reports honoraria, personal fees and research grant funding from Boehringer Ingelheim, Honoraria and personal fees from Roche Pharmaceuticals, Teva, Astra Zeneca, Cipla, fees and stock options from Brainomix. WD-CM reports research grant funding from the National Institute for Health Research and the British Lung Foundation, and honoraria / personal fees from Novartis, Mundipharma, European Conference and Incentive Services and Jazz Pharmaceuticals. ADA is Chief Medical Officer, Santersus AG. PK declares support from Oxford Imunotech for studies of SARS-COV-2 vaccine responses in immune deficient patient populations. AnS reports research grants from Vertex pharmaceuticals, Pfizer and Gilead Sciences and speaker fees from Pfizer and Gilead Sciences. ArS has received honoraria for speaking from Astra Zeneca.

\section{Figures}

\section{Figure 1}

Upregulated systemic immune signature at 3-6 months following severe COVID19: (A) Schematic of Study Design. (B) Principal Component analysis. Each point represents a sample. Colouring indicates COVID19 severity. (C) Proteins upregulated (red) or downregulated (blue) in Severe COVID19 positive patients versus mild COVID19 positive patients at 3-6 months post recovery from acute illness. Linear gradient indicates the effect size. A positive effect size (right side of graph) indicates that an increase in protein level is associated with severe COVID19 cases and a negative gradient (left side of graph) the opposite. P-values from linear mixed models after Benjamini-Hochberg adjustment; significance 
threshold= 5\% FDR; Grey = non-significant. (D) Heatmap showing protein levels. Each column represents a sample ( $\mathrm{n}=46$ severe COVID19 samples, 18 mild COVID19 samples and 17 healthy controls). Each row represents a protein. Differentially expressed proteins between severe COVID19 versus mild COVID19 groups are shown (E) Violin plots showing distribution of selected plasma protein levels at 3-6 months following severe or mild COVID19 and healthy uninfected controls. Boxplots indicate median and interquartile range. $(F) \mathrm{GO}$ biological processes enrichment analysis of differentially expressed proteins. $\star P<0.05 ; * \star P<0.01 ; * \star \star \star * P<0.0001$.

\section{Figure 2}

\section{An upregulated systemic pro-inflammatory neutrophil-associated immune signature characterises post- COVID19 interstitial lung changes.}

(A) Schematic of Study Design (B) Principal Component analysis. Each point represents a sample. Colouring indicates presence or absence of interstitial lung changes. (C) Heatmap showing protein levels. Each column represents a sample $(n=26$ Subjects with interstitial lung changes, $n=20$ with full resolution of lung changes). Each row represents a protein. Differentially expressed proteins between severe COVID19 versus mild COVID19 groups are shown. (D) Proteins upregulated (red) or downregulated (blue) in subjects with interstitial lung changes post recovery. Linear gradient indicates the effect size. A positive effect size (right side of graph) indicates that an increase in protein level is associated with presence of interstitial lung changes and a negative gradient (left side of graph) the opposite. P-values from linear mixed models after Benjamini-Hochberg adjustment; significance threshold= 5\% FDR; Grey $=$ nonsignificant. (E) Violin plots showing distribution of selected plasma protein levels at 3-6 months in subjects with interstitial lung changes versus those without. Boxplots indicate median and inter-quartile range. (F) GO biological processes enrichment analysis of differentially expressed proteins. (G) Correlation between plasma proteins and lung function measurements (\% predicted of forced vital capacity (FVC) and gas transfer factor (TLCO)) (H) Correlation between plasma proteins and extent of

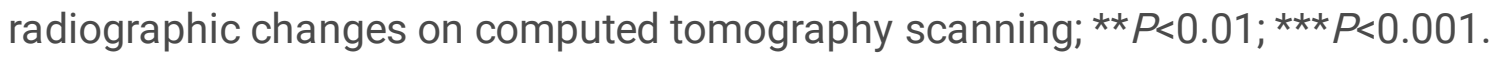

\section{Figure 3}

An upregulated nasal pro-inflammatory and anti-viral immune signature characterises post COVID19 interstitial lung changes. 
(A) Schematic of Study Design (B) Principal Component analysis. Each point represents a sample. Colouring indicates presence or absence of interstitial lung changes. (C) Genes upregulated (right) downregulated (left) in subjects with interstitial lung changes post-recovery. Linear gradient indicates the effect size. A positive effect size (right side of graph) indicates that an increase in gene expression is associated with presence of interstitial lung changes and a negative gradient (left side of graph) the opposite. P-values from linear mixed models after Benjamini-Hochberg adjustment; significance threshold=5\% FDR; (D) Heatmap showing gene expression levels. Each column represents a sample ( $n=16$ Subjects with interstitial lung changes, $n=10$ with full resolution of lung changes). Each row represents a gene. Differentially expressed genes between severe COVID19 versus mild COVID19 groups are shown. (E) Violin plots showing distribution of selected genes at 3-6 months in subjects with interstitial lung changes versus those without. Boxplots indicate median and inter-quartile range. $(F)$ GO biological processes enrichment analysis of differentially expressed genes. (G) Cellular deconvolution analysis of transcriptomic data $(\mathrm{H})$ Correlation between $I L 17 D$ expression and extent of radiographic changes on computed tomography scanning (left graph) and lung function measurements (\% predicted of transfer factor of carbon monoxide (TLCO)); ${ }^{*}<0.05$.
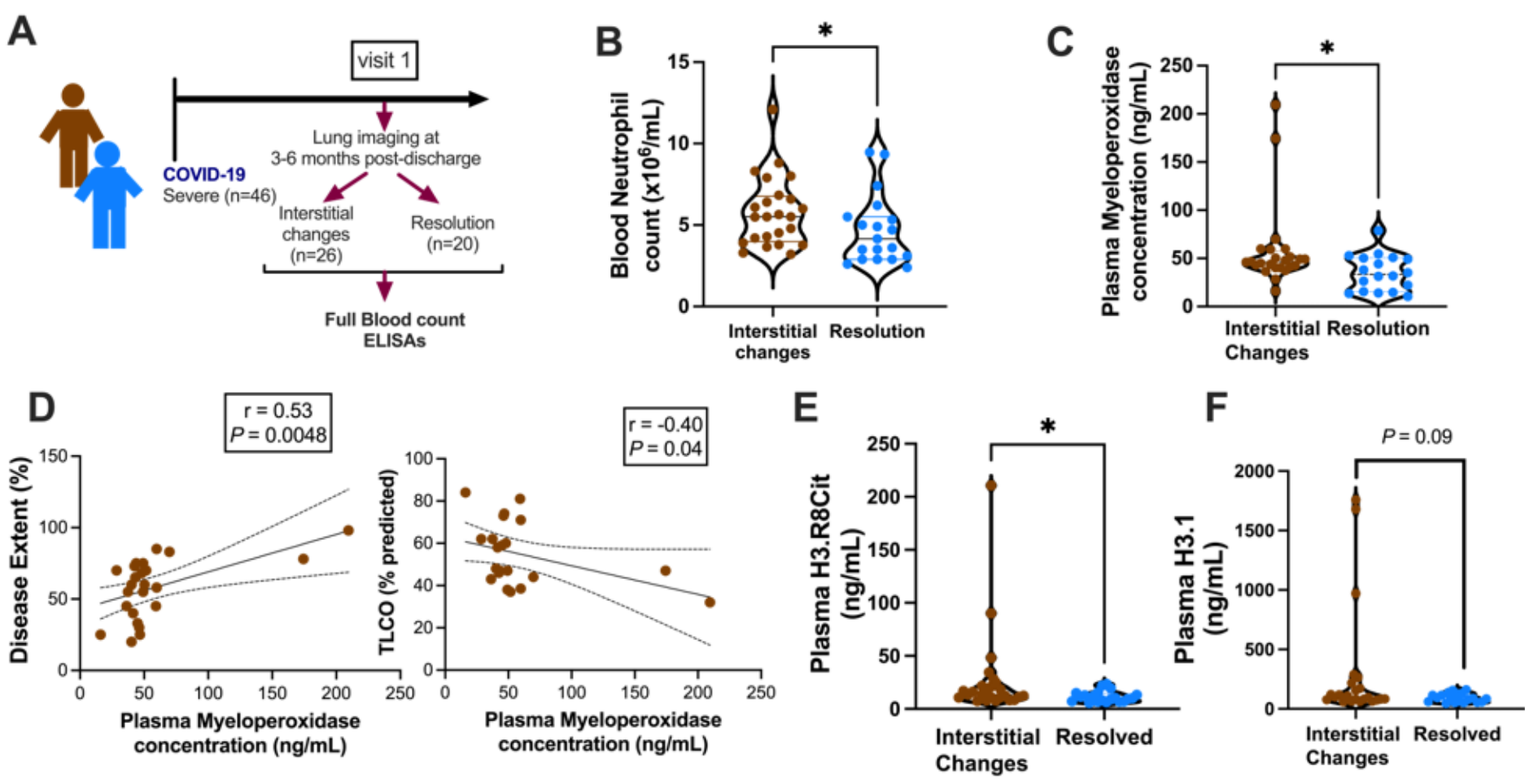

Figure 4

Increased systemic neutrophils, neutrophil protease and NET concentrations are associated with post COVID19 interstitial lung changes. (A) Schematic of Study Design. (B) Total neutrophil counts measured in blood taken at 3-6 months post-discharge from severe COVID19. (C) Plasma myeloperoxidase concentrations quantified by ELISA (D) Correlation between Myeloperoxidase concentrations and extent 
of radiographic changes on computed tomography scanning (left graph) and lung function measurements (\% predicted of transfer factor of carbon monoxide (TLCO). Plasma concentrations of (E) H3.R8 citrullinated nucleosomes and (F) Total intact nucleosomes were measured by H3.1 ELISA. In (B$\mathrm{C})$ and (E-F), boxplots indicate median and inter-quartile range and data analysed by Mann Whitney $U$ test. In (D) data analysed by Spearman's rank correlation test. ${ }^{*} P<0.05$.

\section{Figure 5}

Protein phosphorylation and kinase activities associated with immune cell response are enriched in PBMCs from patients with COVID19 interstitial lung changes. (A) Schematic of Study Design (B) Principal Component analysis. Each point represents a sample and the proportion of the total variance explained by each principal component is shown in parentheses of the plot axes. Colouring indicates presence or absence of interstitial lung changes. (C) Volcano plot of phosphopeptides differentially expressed in the PBMCs of patients with COVID19 ILC vs resolved. Points represent individual phosphopeptides coloured according to the log-odds ratio of being differentially expressed. The dotted red line indicates an arbitrary threshold of a $99 \%$ probability of being differentially expressed (i.e., a log-odds ratio $>4.60$ ). (D) Heatmap showing phosphorylation sites that are differentially regulated $(F D R<0.05)$ between patients with interstitial lung changes vs resolved. Each column represents a sample ( $n=9$ Subjects with ILC, $n=8$ with full resolution of lung changes). Each row represents a phosphopeptide containing 1-4 phosphorylated sites. (E) Kinase network analysis using characteristics computed from the KSEA network results. Points (kinases) are coloured according to their node strength (the sum of weights of links connected to the kinase; ILC vs resolved). Node betweenness (y-axis) represents the degree to which kinases stand between each other (betweenness centrality indicates the control a kinase has over the network); node degree (x-axis) represents the number of connections a kinase has to other kinases. The left panel represents kinases that are more important to maintaining the signalling activity in ILC samples, and the right panel, kinases that are integral to the samples derived from patients with resolved changes.

\section{Figure 6}

Resolution of clinical parameters and immune alterations by 12 months post COVID19. (A) Schematic of Study Design. Time course of (B) FVC (Forced vital capacity), (C) TLCO (transfer factor for carbon monoxide) (D) radiological disease extent and (E) symptom scores as measured by St Georges Respiratory Questionnaire (SGRQ) in subjects with interstitial lung changes versus those full resolution of lung changes showing data at the indicated timepoints post discharge. Plasma concentrations of (F) IL17C (G) CXCL8/IL-8 (H) TNF and (I) myeloperoxidase at the indicated timepoints post discharge. Plasma concentrations of circulating nucleosomes associated with (J) H3R8Cit or (K) Total intact nucleosomes 
measured by H3.1 ELISA at the indicated timepoints post discharge. Data in (B-E) shown as individual datapoints with median indicated by red line, analysed by Wilcoxon rank sum test. Data in (F-J) analysed by Mann Whitney $U$ test and data compares interstitial changes with resolution group. ${ }^{\star} P<0.05$, $\star \star \star * P<0.001, * \star \star \star P<0.0001$.

\section{Supplementary Files}

This is a list of supplementary files associated with this preprint. Click to download.

- FINALVERSIONSupplementaryFiguresNatureMed.docx 